\title{
THE INFLUENCE OF THE GEOMETRICAL PARAMETERS FOR STRESS DISTRIBUTION IN WIRE RACEWAY SLEWING BEARING
}

\begin{abstract}
This paper presents the current study of the distribution of stresses for fourpoint contact wire race ball bearing. The main aim of this article is to define the most important geometrical rules in a wire-race bearing. The results for bearings of different geometrical parameters are presented. In the study, one also estimates the distribution of internal pressure in particular bearing elements.
\end{abstract}

\section{Introduction}

The wire-race slewing bearing is a kind of slewing bearing in which a wire plays the role of raceway. The rings can be made of light metals, like aluminum or new materials, like composites. These bearings can be used in applications where lightweight and inertia are important, such as aircraft, robotics and medical aperture [1]. Wire-race slewing bearing are also backlash-free. Another advantage are replaceable raceways, thanks to which the maintenance of big machines (like excavators) is much easier.

In these bearings, there are two contact pairs: ball-wire and wire-ring. The ballwire contact pair is an example of a Hertz contact with elliptical contact surface. The wire-ring contact is a classic contact, with larger contact area. Due to the lack of literature on these kinds of bearings, the design rules for wire bearings are not properly defined. The future expectations for these kinds of bearings are: further reduction of weight, by using composites material, and improvement of reliability.

An example of a cross section of wire-race slewing bearing is presented below (Fig. 1). In the wire-race bearing, the load is usually carried from external components through balls or rollers and wires in four contact points to the ring and the rest of structure. The estimation of the rings deformations is crucial in predicting

\footnotetext{
${ }^{1}$ Wroclaw University of Science and Technology, Wybrzeże Wyspiańskiego 27, 50-370 Wroclaw, Poland.Emails: dominik.gunia@pwr.edu.pl,tadeusz.smolnicki@pwr.edu.pl
} 


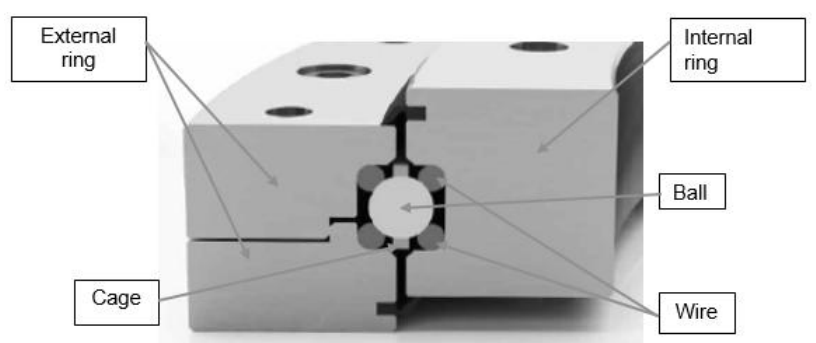

Fig. 1. Section of wire raceway bearing [1]

the life of bearing. The cross sections of wires can have different shapes, but most often the circular cross sections is used. [2-9].

To calculate load bearing capacity, one can use analytical methods, such as the Ohnrich's method, or the Matthias's method, but these method rely on certain assumptions. The use of the finite element method (FEM) allows for the contact analysis and the analysis of the distribution of pressure for particular rolling elements. The results of simulations allow one to determinate certain characteristics (e.g., stiffness characteristics of the ball-wire), and allow for making the assumptions in development of alternative four-point bearing models [2-10].

The aim of this study is to estimate the load distribution, pressure distribution and stress distribution in particular pairs, and to determine the most loaded points and characteristics describing stiffness of kinematic pairs, which can be used in development of an alternative four-point contact bearing model.

\section{Elastic contact - Hertz theory}

The Hertz theory is a classical contact theory used in analysis of elastic bodies. The main assumptions of this method are as following [2, 6-9]:

1. The size of contact is small in comparison with the radii of contacting bodies.

2. The yield limit cannot be exceeded, the bodies can deform only in the elastic range of material property.

3. The load is perpendicular to the contact surface and the contact is frictionless.

4. Geometrical imperfections of surfaces are not taken into account.

In this theory, the contact that occurs between the bodies is only a mathematical contact (point contact and line contact). The contact changes its shape from a point contact to an ellipse, due to deformation of the bodies. The curvature function of the bodies can be calculated as follows [2, 6-9]:

$$
\begin{gathered}
F(\rho)=\frac{\left(\rho_{11}-\rho_{12}\right)+\left(\rho_{21}-\rho_{22}\right)}{\sum \rho} \\
\sum \rho=\rho_{11}+\rho_{12}+\rho_{21}+\rho_{22}
\end{gathered}
$$


In these equation, $\rho$ is principal curvature of the contact object and it is defined as a reciprocal of each radius in the bodies, respectively, with sign conventionpositive for convex surfaces, negative for concave. Assuming material properties, like Young's modulus of the bodies as a $E_{1}$ and $E_{2}$, and Poisson's ratio as $\mu_{1}$ and $\mu_{2}$, one can calculate the semi-axes of the contact ellipse as follows $[2,6-9,11]$ :

$$
\begin{aligned}
& a=a^{*}\left[\frac{3 Q}{2 \sum \rho}\left(\frac{1-\mu_{1}^{2}}{E_{1}}-\frac{1-\mu_{2}^{2}}{E_{2}}\right)\right]^{\frac{1}{3}} \\
& b=b^{*}\left[\frac{3 Q}{2 \sum \rho}\left(\frac{1-\mu_{1}^{2}}{E_{1}}-\frac{1-\mu_{2}^{2}}{E_{2}}\right)\right]^{\frac{1}{3}}
\end{aligned}
$$

Maximum contact pressure can be calculated as follows $[2,6-9,11]$ :

$$
p_{\max }=\frac{1}{2 \pi a^{*} b^{*}}\left[\frac{3 Q\left(\sum \rho\right)^{2}}{\left(\frac{1-\mu_{1}^{2}}{E_{1}}-\frac{1-\mu_{2}^{2}}{E_{2}}\right)^{2}}\right]^{\frac{1}{3}}
$$

Is these formulas, $a^{*}$ and $b^{*}$ are the coefficients of axes of the contact ellipse $[2,6-9,11]$.

\section{Computational model}

\subsection{Finite element model}

In order to simulate FEA phenomena occurring in a wire-race bearing, four bearing models with different geometric parameters are built. Furthermore, in order to shorten calculation time of the simulation, cyclic symmetry and mirror symmetry assumption is used, i.e., only $1 / 4$ of a ball and segments of the rings and wires are modeled.. In the contact area, first-order HEXA (C3D8R) elements are used, element sizes in the contact ball-wire pair are $0.2 \%$ of ball diameter, in the contact wire-ring pair the element size could be bigger due to the model size and calculation time. Whereas, in other contact areas, first-order TETRA (C3D4) elements are used. TETRA elements are connected to HEXA elements by a linear contact "TIE". Fig. 2 shows one of the bearing models, where white arrows symbolize the direction of kinematic extortion. The models consist usually of 700 000-800 000 elements and have 2100 000-2 400000 degrees of freedom. The mesh size is chosen according to literature, hence mesh convergence is not performed $[2,3,12,13]$.

The diameter of balls (d) is in the range from $14.288[\mathrm{~mm}]$ to $30[\mathrm{~mm}]$, the diameter of wire $\left(d_{w}\right)$ is $10[\mathrm{~mm}]$, the osculation ratio $(s)$ is equal to 0.96 and 0.98. The models are constrained as follows: the wire, the ball and the ring are 


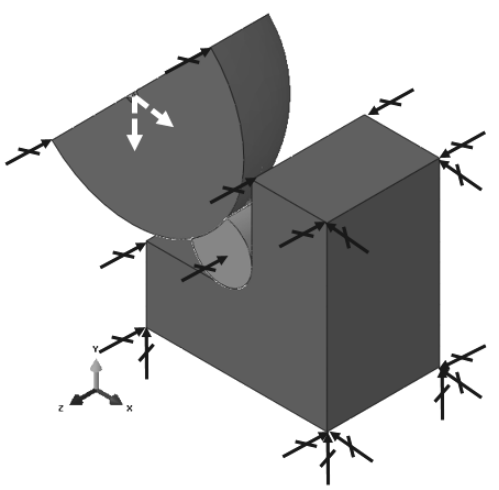

Fig. 2. Bearing model used in simulation with fixed displacement and direction of kinematic extortion

constrained by symmetry bonding (plane XY), and the ring is constrained also in YZ and ZX plane. (Fig. 2).

In the models, contact areas have a friction coefficient equal to $f=0.05$. In the ball-wire contact pair, the master surface is the ball surface, the slave is the wire raceway, and in the next contact pair the master surface is the ring surface and the slave is the wire. The contacts are based on "Surface to surface" discretization method with finite sliding contact formulation. In normal direction, hard contact with the Augmented Lagrange algorithm is used. In order to obtain an easier convergence, one uses automatic stabilization. This stabilization is based on dissipated energy fraction equal to 0.0002 , and an adaptive stabilization with max ratio equal to 0.05 is added. The initial step size is equal to 0.0001 and could change in the range from 0.00001 to 1 . The full Newton method is used in simulations [5, 12-17].

Simulations are done in the linear range of material behavior, and the material constants are:

- ball-steel: $E=200 \mathrm{GPa}, v=0.3$,

- wire-steel: $E=200 \mathrm{GPa}, v=0.3$,

- ring-aluminum: $E=200 \mathrm{GPa}, v=0.33$.

The simulation is based on displacement of a reference point inside a ball with the resultant distance of 0.1 [mm] in the XY-plane. Next, Huber-Mises stresses variability is assumed for the wire and the ball in the Bielajew point and at the contact edge, whereas for the ring-only at the contact edge. The pressure charts are made for the contact edge and for the central point in the contact area. The distribution of particular deformation of the elements is also checked. The results are presented in the function of ball load ( $\mathrm{pw}$ ), while the ball load is defined as:

$$
p_{w}=\frac{F}{d^{2}}
$$

where: $F$ - force acting for ball, $d$ - ball diameter. 
An important parameter for wire-race bearings is the osculation ratio $(s)$. The simulations were run for two different osculation ratios ( 0.96 and 0.98$)$. It is defined as:

$$
s=\frac{d}{2 r_{b}}
$$

where: $d$ - ball diameter, $r_{b}$ - wire raceway radius.

For the purpose of this simulations, a new factor is defined, which is the fill factor of raceway $(k)$. The calculations are performed for three values of this factor $(0,382 ; 0,725 ; 0,987)$. The factor it is defined as:

$$
k=\frac{c_{w}}{d_{w}}
$$

where: $c_{w}$ - chord of raceway, $d_{w}$ - wire diameter.

\subsection{Results and discussion}

For the displacement in which the contact occurs on the edge of a wire, the percentage distribution of deformation is calculated for each of the elements of bearings. The ball deforms on average by $49 \%$ of the total bearing deformation. The wire accounts for $39 \%$ of deformation, and the ring accounts for $12 \%$.

The relationship between maximum von Mises stress in the wires in the Bielajew's point and the ball load is presented in Fig. 3. Von Mises stress reaches a value of $\sigma_{H M}=3500[\mathrm{MPa}]$ for ball diameter $14.288[\mathrm{~mm}]$ and the osculation ratio equals 0.96 . According to literature, higher osculation ratio is due to a lower stress, which is observed in this figure, in which the stresses for ball diameter of $20[\mathrm{~mm}]$ and $30[\mathrm{~mm}]$ are similar. Higher stress for ball diameter equal to $20[\mathrm{~mm}]$ might be an effect of a difference in another geometrical factors. Stress distribution is nonlinear, the higher osculation ratio is due to different shape of stress area. The

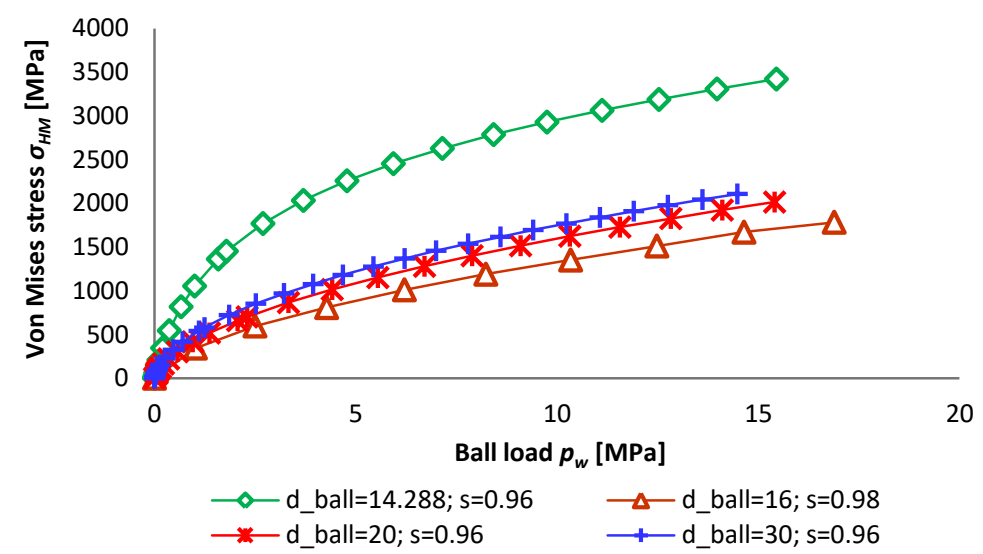

Fig. 3. Comparison of maximum Von Mises stresses in wires, [MPa] 
stress in the wire is higher by approximately $9 \%$ than the stress in the ball, and because its distribution is similar, only results for the wire are presented $[2,14]$.

The next figure (Fig. 4) presents the maximum von Mises stress [MPa] on the contact edge in the wire. The simulations are performed with no fillet on the wire edge, hence in this place there is a structural notch, which can also cause an effect of singularity, however, higher stresses are expected in this area. For a low value of ball loads, the stresses are also low, because the ball is not in contact with the edge. After the contact occurs, an increase in stress is observed. In a properly working bearing, the contact between the ball and the wire edge should be avoided, however, this scenario should be analyzed. Edge correction (e.g., fillet or logarithmical correction) should decrease the stress at the edge.

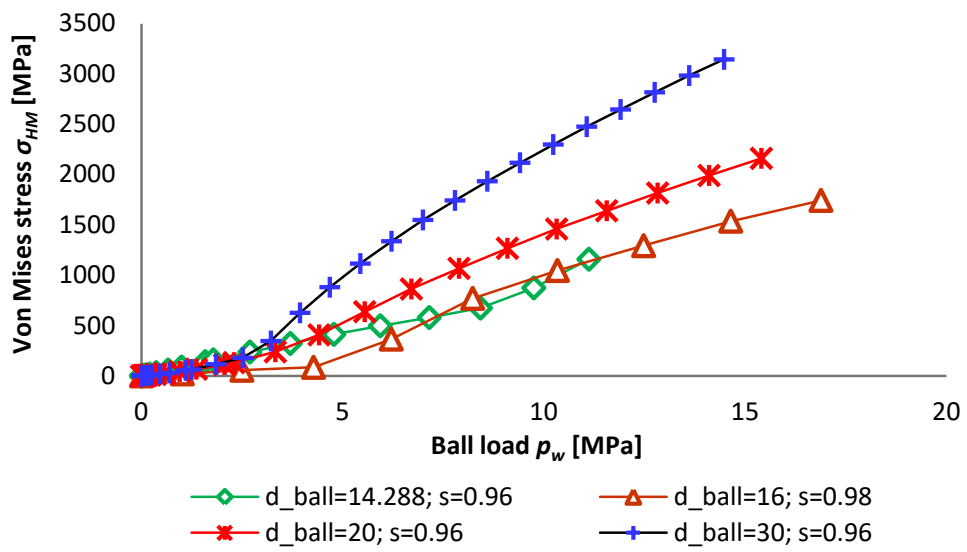

Fig. 4. Comparison of maximum Von Mises stresses on the contact edge in wires, [MPa]

The results for the wire-ring contact pair indicate that, in this pair, stress distribution is linear, due to the lack of the Hertz stress (Fig. 5 and Fig. 6). Stress levels are significantly lower than those in the ball-wire contact pair; maximum stress in the notch at the end of the contact surface is $760[\mathrm{MPa}]$, in the middle of the contact surfaces the stress is, on average, lower than $30 \%$.

The growth of stresses in the rings is linear, whereas in the balls and wires it is nonlinear. In the case of rings, it is an effect of lack of Hertz stress, therefore, in the rings the stress is lower, hence materials with lower yield limit could be used, such as aluminum. In the balls and wires, a nonlinear distribution is a result of the Hertz stress and ellipsoidal shape of the contact stress. In the rings, high contact stresses are observed at the end of the contact area. It can be due to the influence of the notch, or just spurious results due to numerical errors in the contact formulations. However, if there is any yielding in the rings, it can have an influence on clearance of parts, which may cause fatigue damage.

According to literature [4], maximum Hertz contact pressure can reach 42004600 [MPa] $[4,12]$. An example of the contact pressure plot and Von Mises stress 


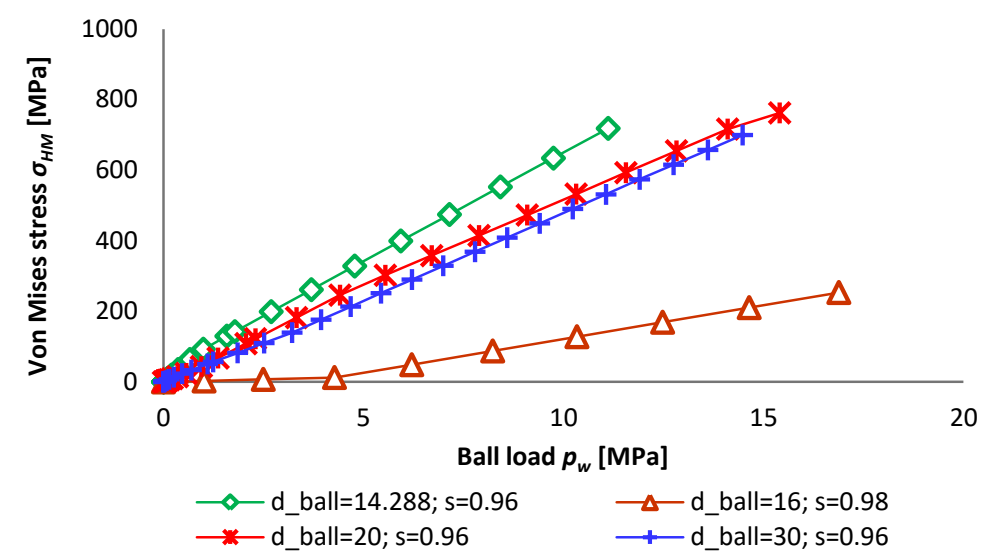

Fig. 5. Comparison of maximum Von Mises stress on the contact end in the pair wire-ring

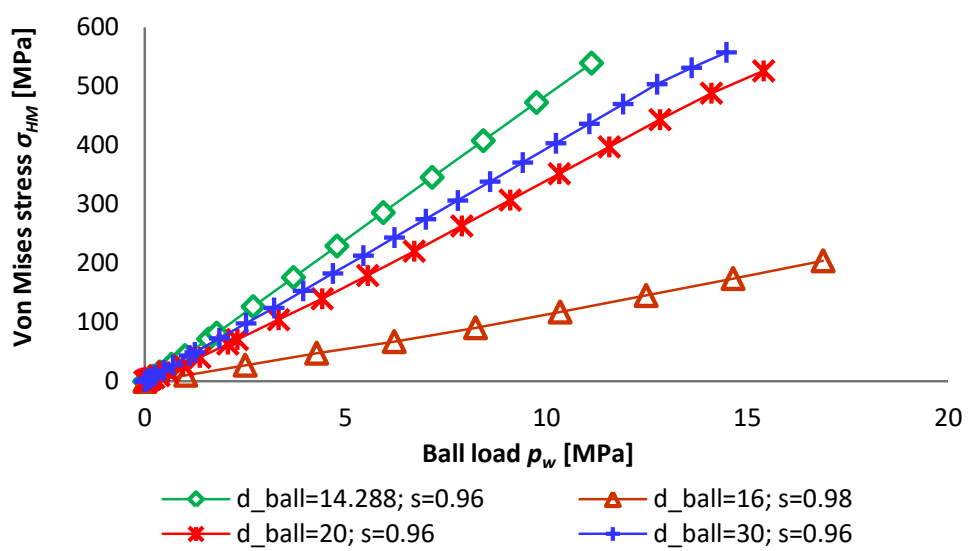

Fig. 6. Comparison of maximum Von Mises stress in the middle of the contact pair wire-ring

is presented in Fig. 7. The maximum contact pressure in the middle of the contact in this case is $p_{\max }=1760$ [MPa]. This plot is for ball load $p_{w}=3.2[\mathrm{MPa}$. For this value of a ball load, the contact occurs on the wire edge. An ellipsoidal shape of contact pressure is visible. The contact pressure plot in the case of wire is analogical. In the second contact pair, the wire-ring contact pressure is significantly lower in comparison to the ball-wire contact pair.

The pressure in the middle of a contact reaches the highest value only when the ball does not reach a wire edge. For the contact of ball with a wire edge, one can observe a significant increase in the stress and in the contact pressure. In the wire-ring contact pair, stresses in the middle of the contact reach 550 [MPa], while at the end of contact surfaces this stress can grow by $50 \%$, hence it is necessary to correct these contact surfaces. Examples of results are presented in Fig. 8 (for the ball-wire contact pair) and Fig. 9 (for the wire-ring contact pair). 

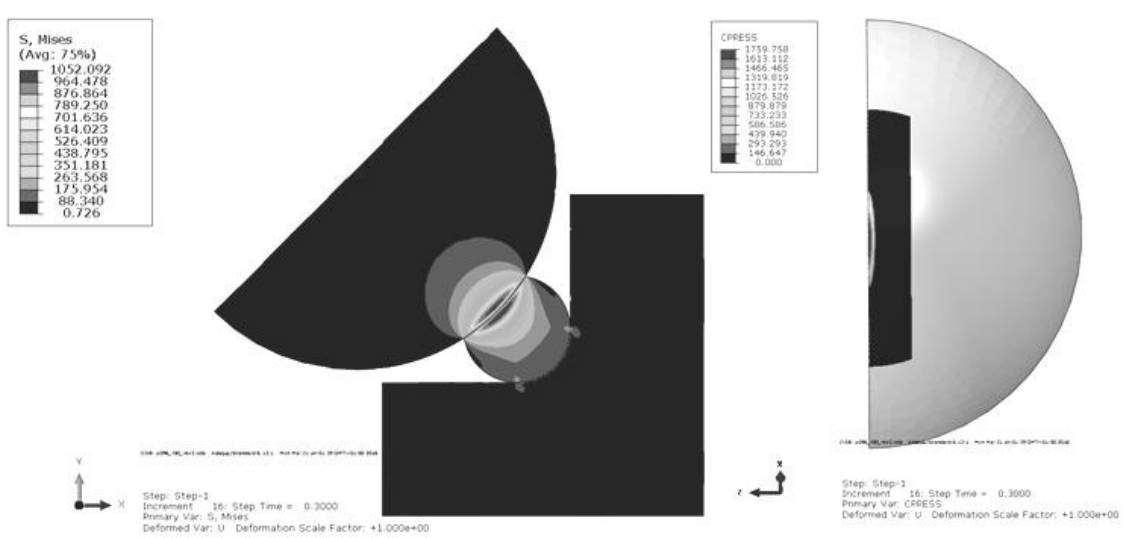

Fig. 7. Von Mises stress and contact pressure in ball

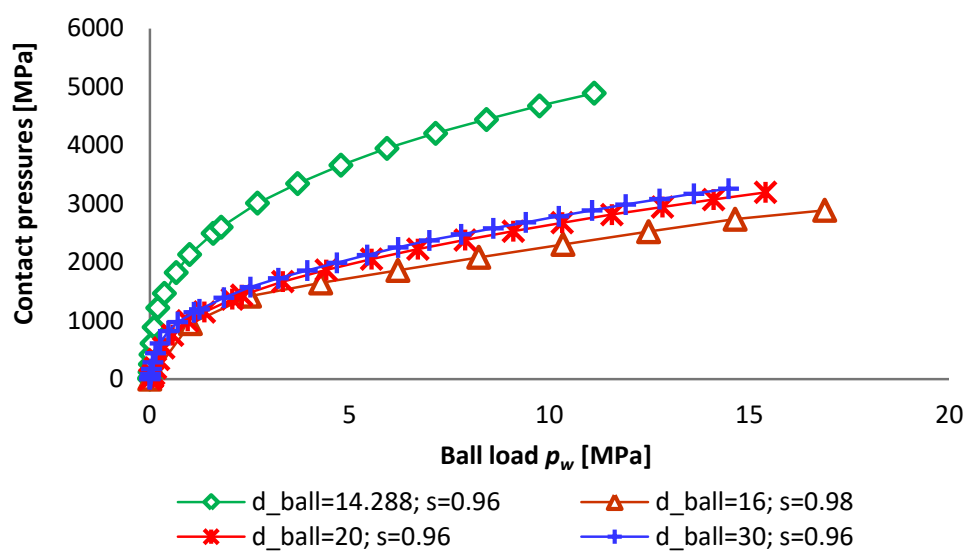

Fig. 8. Comparison of contact pressures in the ball-wire contact pair

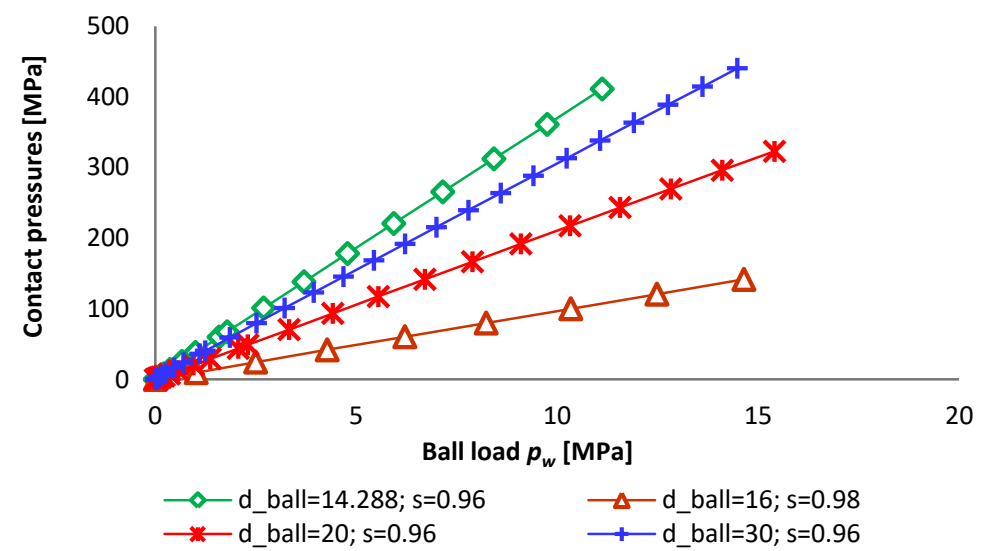

Fig. 9. Comparison of contact pressures in the wire-ring contact pair 
Comparison between analytical results and finite element analysis results of contact pressure and stress have shown that analytical method gives comparable results for a higher value of ball diameter (Table 1). For lower balls diameters, discrepancy between the results is very high. The analytical method of stress calculation can be treated as a preliminary step for further calculation, however, the results of finite element analysis are more accurate [2]. FEA simulations allows us to include more phenomena, which are restricted for analytical models [2].

Table 1

\begin{tabular}{|c|c|c|c|c|c|}
\cline { 3 - 6 } \multicolumn{2}{c|}{} & \multicolumn{2}{c|}{ Analytical } & \multicolumn{2}{c|}{ FEA simulations } \\
\hline Bearing model & $\begin{array}{c}\text { Force } \\
{[\mathrm{N}]}\end{array}$ & $\begin{array}{c}\text { Pressure } \\
{[\mathrm{MPa}]}\end{array}$ & $\begin{array}{c}\text { Von Mises } \\
\text { stress [MPa] }\end{array}$ & $\begin{array}{c}\text { Pressure } \\
{[\mathrm{MPa}]}\end{array}$ & $\begin{array}{c}\text { Von Mises } \\
\text { stress [MPa] }\end{array}$ \\
\hline$d=14, s=0.96$ & 200 & 880.44 & 545.87 & 2132.2 & 1053.95 \\
\hline$d=16, s=0.98$ & 640 & 1038.00 & 643.56 & 1419.57 & 597.36 \\
\hline$d=20, s=0.96$ & 1300 & 1003.55 & 622.20 & 1668.51 & 864.66 \\
\hline$d=30, s=0.96$ & 2200 & 1536.34 & 952.53 & 1573.74 & 852.07 \\
\hline
\end{tabular}

The raceway fill factor is created to explain the difference in stress results, e.g., the fact that Von Mises stress in the Bielajew point for the bearing with ball diameter equal to 14.288 [mm] is $70 \%$ higher in comparison to the rest of results. The results of stress distribution in the wire race bearing for different values of raceway fill factor are presented in Fig. 10. Simulations have been performed for equal geometrical parameters, such as ball diameter and osculation ratio. For a low value of $\mathrm{k}$, the contact between the ball and the wire edge occurred for low values of ball load. At higher values of raceway fill factor, the stress at the Bielajew's point may decrease.

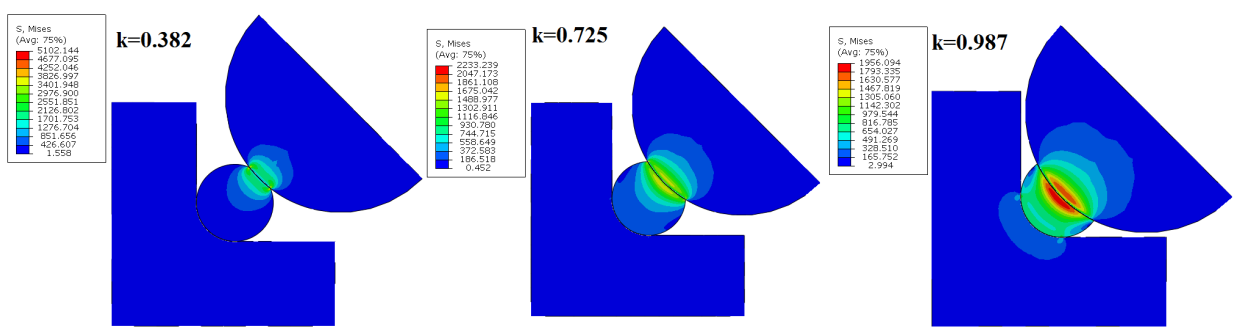

Fig. 10. Comparison of stress distribution for different values of raceway fill factor $(k)$ in wire race bearing

The next figures (Fig. 11 and Fig. 12) show stress distribution at the Bielajew's point and on wire edge. For a higher value of fill factor, the Hertz stress can decrease (10\% difference at the Bielajew's point,almost $300 \%$ for Von Mises stress 
at the edge). On the boundary of contact edge, stress distribution changes from three-axis compression to two-axis state of load. This situation is observed for higher values of ball load and is dangerous, because the stress can reach yield limit. Stress distribution at the contact edge is nonlinear, however, different types of nonlinearity can be observed for lower and higher values of the raceway fill factor. For $k=0.382$, the distribution is initially nonlinear, for higher values of $k$, the distribution is initially almost linear and then it becomes nonlinear. This phenomenon can be explained by the fact that, for higher values of $k$, the ball can deform much more.

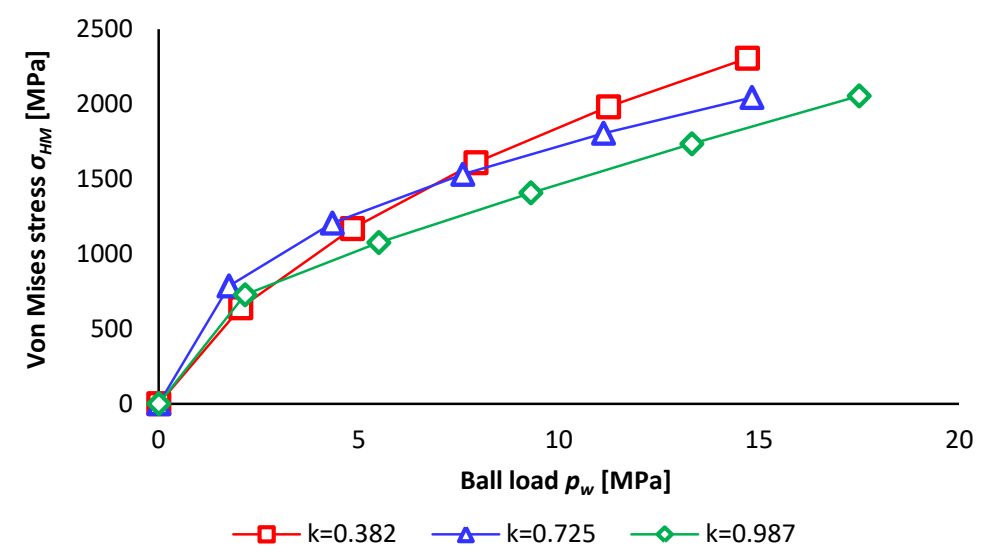

Fig. 11. Comparison of maximum Von Mises stress at the Bielajew's point for different values of raceway fill factor

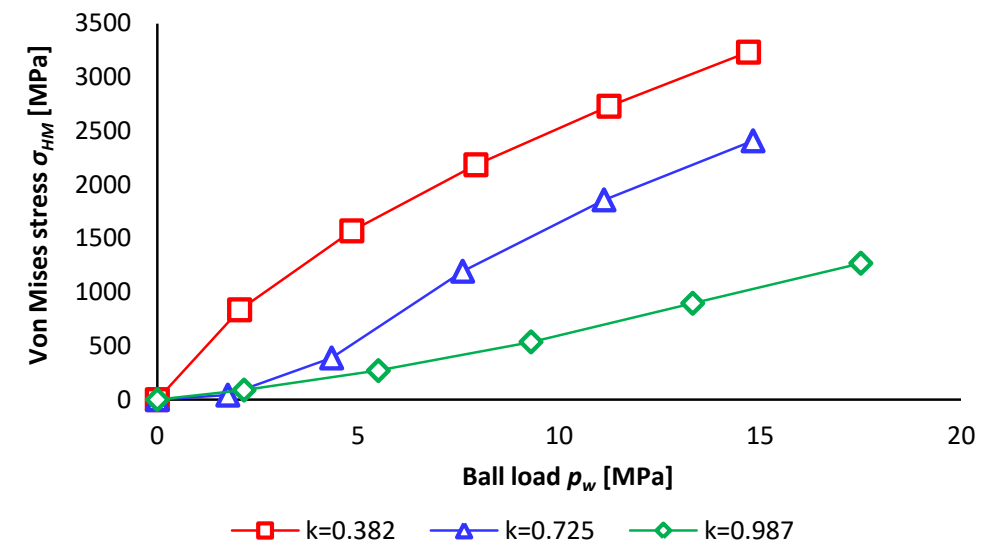

Fig. 12. Comparison of maximum Von Mises stress on the wire edge for different values of raceway fill factor 


\section{Conclusions}

Four-point contact wire raceway bearings can be analyzed in a similar way like normal slewing bearing. In the wire-ring type of pair, the maximum contact stresses occurs at the edge of the contact (the contact stresses are about 50\% higher than those in the middle of the contact), therefore it is essential to introduce a geometrical correction for this area.

The next critical point is the contact edge in the ball-wire pair, where it is necessary to check this contact area according to maximum Von Mises stress and the contact stress. It is also important to make a geometrical correction for the contact edge, for example, curvature correction (fillet), or logarithmic correction. However, well-designed wire race bearing should not work in this range of load.

The bearings with higher osculation ratio can reach a higher value of stresses, hence they can be greatly loaded. However, the contact between wire edge and ball appears for a lower value of ball load.

In well-designed wire-raceway slewing bearing, the most loaded points are the Bielajew point (the highest Von Mises stress) and the middle of contact in the ball-wire pair (the highest contact stress). This stresses can be calculated with an analytical method, however, for a lower ball diameter the calculation error can be significant. Finite element analysis is suggested instead as a reasonable method for stress calculation.

Wire raceway bearings can be an alternative to classical slewing bearing in the cases where weight is the priority. The most loaded parts are the balls and the wire, hence the ring can be made of materials of lower material strength (like aluminum).

The raceway fill factor is significant, it can reduce the load by $10 \%$ at the Bielajew's point and by almost $300 \%$ at the point on the wire edge. However, for a high value of this factor, the results of these calculations may not be accurate, due to the lack of plasticity in the material model.

The results of this kind of analyses (e.g., element stiffness) can be used in simulations of a full model of bearing with methodology suggested in literature [2]. Simulations with the use of virtual elements make it possible to evaluate the behavior of a full slewing bearing model with a real carrying structure. This is a huge advantage, especially in the case of large-diameter bearings.

\section{Manuscript received by Editorial Board, February 11, 2017; final version, May 03, 2017.}

\section{References}

[1] Franke GmbH. Drahtwaelzlager, 2014. (in German).

[2] T. Solnicki. Large-diameter rolling bearings. Global and local problems. Publishing House of Wroclaw University of Science and Technology, 2013. (in Polish). 
[3] E. Rusiński, J. Czmochowski, and T. Smolnicki. Advanced Finite Elements Method in carrying structures. Publishing House of Wroclaw University of Science and Technology, 2000. (in Polish).

[4] L. Kania. Local problems in numerical modeling of slewing bearings. Publishing House of Czestochowa University of Technology, 2012. (in Polish).

[5] L. Kania. Analysis of inner load in slewing bearings. Publishing House of Czestochowa University of Technology, 2005. (in Polish).

[6] H. Hertz. On the contact of rigid elastic solids and on hardness. In Miscellaneous Papers, pages 163-183. MacMillan, London, 1896.

[7] G. Lundberg and H. Sjovall. Stress and deformation in elastic contacts. Gothenburg: Institution of Theory of Elasticity and Strength of Materials, Chalmers University of Technology, 1958.

[8] A. Palmgren. Ball and roller bearing engineering. SKF Industries, Philadelphia, 3rd edition, 1959.

[9] N.M. Bielajew. Computation of maximal stresses obtained from formulas for pressure in bodies in contact. In Bull. Eng. Ways Commun. Leningrad, 1929.

[10] A. Daidié, Z. Chaib, and A. Ghosn. 3D simplified finite elements analysis of load and contact angle in a slewing ball bearing. Journal of Mechanical Design, 130(8):082601-082601-8, 2008. doi: 10.1115/1.2918915.

[11] You Hui-yuan, Zhu Chun-xi, and Li Wu-Xing. Contact analysis on large negative clearance four-point contact ball bearing. Procedia Engineering, 37:174-178, 2012. doi: 10.1016/j.proeng.2012.04.222.

[12] Abaqus/CAE User's Manual.

[13] P. Göncz, M. Ulbin, and S. Glodež. Computational assessment of the allowable static contact loading of a roller-slewing bearing's case-hardened raceway. International Journal of Mechanical Sciences, 94:174-184, May 2015. doi: 10.1016/j.jjmecsci.2015.03.006.

[14] R. Pandiyarajan, M.S. Starvin, and K.C. Ganesh. Contact stress distribution of large diameter ball bearing using Hertzian elliptical contact theory. Procedia Engineering, 38:264-269, 2012. doi: 10.1016/j.proeng.2012.06.034.

[15] R. Lostado, R.F. Martinez, and B.J. Mac Donald. Determination of the contact stresses in double-row tapered roller bearings using the finite element method, experimental analysis and analytical models. Journal of Mechanical Science and Technology, 29(11):4645-4656, 2015. doi: 10.1007/s12206-015-1010-4.

[16] S.W. Hong and V.C. Tong. Rolling-element bearing modeling: A review. International Journal of Precision Engineering and Manufacturing, 17(12):1729-1749, 2016. doi: 10.1007/s12541016-0200-z.

[17] I. Heras, J. Aguirrebeitia, and M. Abasolo. Friction torque in four contact point slewing bearings: Effect of manufacturing errors and ring stiffness. Mechanism and Machine Theory, 112:145-154, June 2017. doi: 10.1016/j.mechmachtheory.2017.02.009. 